\title{
Having the guts to grow
}

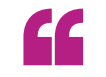

\section{stem cell}

activity can

be regulated

... in response

to dietary

change.

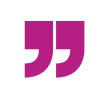

Stem cell activity functions to maintain tissue homeostasis in adult organs. Organs and tissues must also be capable of remodelling, growing or shrinking in response to environmental changes, but how these adaptive mechanisms are regulated is unknown. Bilder and colleagues now show that intestinal stem cells in the adult Drosophila melanogaster midgut drive tissue growth in response to food abundance by increasing their division rates and predominantly dividing symmetrically.

The authors first observed that 4-day-old adult flies that had fed continuously had larger midguts than their fasting counterparts, although the shape of the midgut was the same. Organ enlargement was due to an increase (by $\sim 300 \%$ ) in the total number of midgut cells.

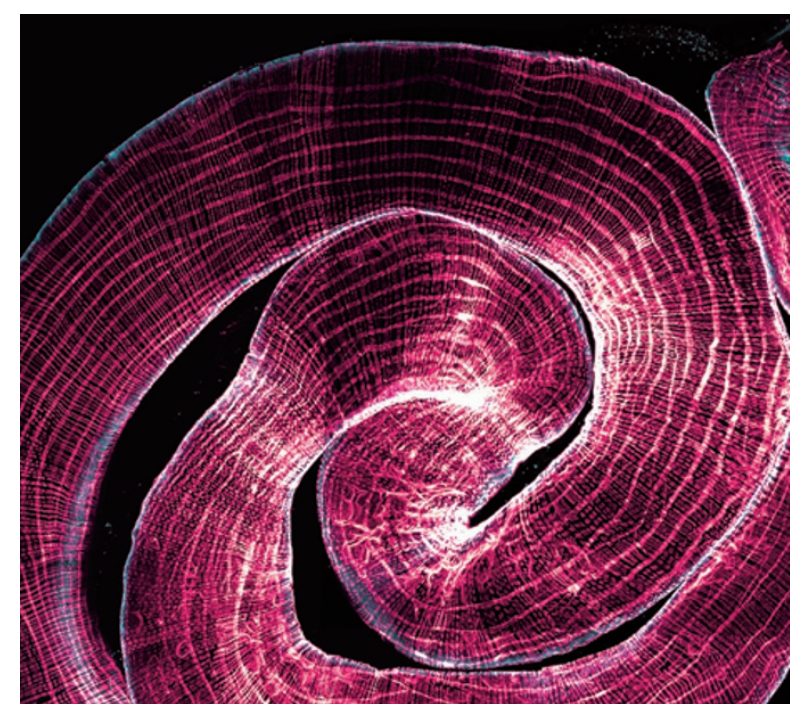

Visceral muscle (pink) surrounding the fly intestinal epithelium secretes insulin upon feeding to activate intestinal stem cells and induce organ growth. Image courtesy of D. Bilder, University of California, Berkeley, USA.
But what is the source of these additional cells? Stem cells are the only dividing cells in the gut, and their immediate progeny are immature cells called enteroblasts. Because the number of enteroblasts in the midgut increased upon feeding, tissue growth might be dependent on stem cell activation. However, higher enteroblast numbers did not account for the total increase in midgut cells. This, together with the observation that there were more stem cells in the guts of fed flies, suggested that stem cell symmetric divisions might also contribute to tissue growth.

To test this hypothesis, the authors monitored symmetric and asymmetric divisions using a genetic marking system that allows the two daughters of a stem cell, and their progeny, to be differentially and permanently labelled. Interestingly, the majority of stem cells (68\%) were found to divide symmetrically rather than asymmetrically in growing guts; this ratio was inverted in the guts of fasting flies. Thus, the predominance of symmetric division appears to increase the total number of cells for adaptive growth.

Next, the authors sought to determine the signals that induce proliferation and growth in the midgut. They found that local levels of Insulin-like peptide 3 (ILP3), a protein previously shown to be expressed in midgut visceral muscle, increased with kinetics that coincided with the dynamics of stem cell proliferation and organ growth. In addition, knockdown of Ilp3 reduced stem and total cell numbers in the guts of fed flies, whereas ILP3 overexpression had the opposite effect in the guts of fasting flies. This suggests that local ILP3 promotes feeding-induced stem cell proliferation and organ growth. Furthermore, the authors found that stem cell proliferation was dependent on the insulin receptor INR.

Finally, the authors discovered that the increase in stem cell activity and the resulting tissue growth are reversible. Adult midguts decreased in size (and total cell number) after fasting, owing to reduced stem cell activity combined with an increase in apoptosis.

These results show that stem cell activity can be regulated to ensure the reversible, adaptive non-homeostatic growth of the midgut in response to dietary change.

Kim Baumann

ORIGINAL RESEARCH PAPER O'Brien, L. E. et $a$. Altered modes of stem cell division drive adaptive intestinal growth. Cell 147, 603-614 (2011) 